\title{
Superorbital modulation of X-ray emission from gamma-ray binary LSI +61303
}

\author{
Maria Chernyakova ${ }^{* a b}$, Andrii Neronov ${ }^{c}$, Sergey Molkov ${ }^{d}$, Denys Malyshev ${ }^{e}$, \\ Alexander Lutovinov ${ }^{d}$, Guy Pooley ${ }^{f}$ \\ ${ }^{a}$ Dublin City University, Glasnevin, Dublin 9, Ireland \\ ${ }^{b}$ DIAS, Fitzwiliam Place 31, Dublin 2, Ireland \\ c ISDC Data Center for Astrophysics, Chemin d'Ecogia 16, 1290 Versoix, Switzerland \\ ${ }^{d}$ Space Research Institute (IKI), 84/32 Profsoyuznaya Str, Moscow 117997, Russia \\ ${ }^{e}$ Bogolyubov Institute for Theoretical Physics, 14-b Metrolohichna street, Kiev, 03680, Ukraine \\ ${ }^{f}$ Astrophysics, Cavendish Laboratory, Cambridge CB3 OHE, UK \\ E-mail: masha.chernyakova@dcu.ie
}

\begin{abstract}
We report the discovery of correlated modulation of X-ray and radio emission properties of the gamma-ray binary LSI +61 303 on a superorbital 4.6 year time scale. Using systematic multiyear monitoring of the system by RXTE we show that the orbital phase of X-ray flares from the source varies from $\phi_{X} \simeq 0.35$ to $\phi_{X} \simeq 0.75$ on the superorbital time scale. Simultaneous radio observations show that periodic radio flares always lag the $\mathrm{X}$-ray flare by $\Delta \phi_{X-R} \simeq 0.2$. We propose that the constant phase lag corresponds to the time of flight of the high-energy particle filled plasma blobs from inside the binary to the radio emission region at the distance $\sim 10$ times the binary separation distance. We put forward a hypothesis that the X-ray bursts correspond to the moments of formation of plasma blobs inside the binary system.
\end{abstract}

An INTEGRAL view of the high-energy sky (the first 10 years) - 9th INTEGRAL Workshop and celebration of the 10th anniversary of the launch

15-19 October 2012

Bibliotheque Nationale de France, Paris, France

${ }^{*}$ Speaker. 


\section{Introduction}

LSI +61 303 is one of several high-mass $\mathrm{X}$-ray binaries visible from radio up to TeV. From radio observations we know that LSI +61303 is variable on different time scales. The orbital period is $P_{\text {orb }}=26.496 \mathrm{~d}$ [5]. The superorbital period is $P_{s o}=1667 \mathrm{~d}$ [5]. The zero orbital and superorbital phase $\Phi=0$ is $T_{0, s o}=2443366.775+k P_{o r b} / s o$. The radio signal could not be used to trace the outflow down to the production site inside the binary orbit, because of the free-free absorption in the dense stellar wind environment, e.g. [13]. To understand the nature of the high-energy particle carrying outflow one has to use complementary high-energy data in X-ray and/or $\gamma$-ray bands.

The X-ray and $\gamma$-ray emission from the system is known to be variable on the orbital time scale, e.g. [2] and references therein. A study of the orbital modulation of the X-ray and $\gamma$-ray signal was missing up to recently due to the absence of systematic monitoring of the source on many orbit (year) time scale. Such a monitoring has recently became possible in the GeV $\gamma$-ray band with the start of operation of the Large Area Telescope (LAT) on board of Fermi satellite [1]. In the X-ray band, a dedicated multi-year monitoring campaign was done with RXTE [11]. As a result, both the average periodic modulation pattern and orbit-to-orbit variations of X-ray and $\gamma$-ray emission from the source were established.

In this paper we discuss our study of the changes in the behaviour of the X-ray and $\gamma$-ray emission on superorbital time scale, as observed by RXTE, INTEGRAL and Fermi, please see this paper for the details of data analysis. The X-ray/ $\gamma$-ray data are complemented by the contemporaneous radio monitoring data. The comparison of the X-ray behaviour with the historical radio data is given in [6].

\section{Available data and results}

In our analysis we consider radio, X-ray and $\gamma$-ray data on LSI +61 303 collected over the period from 2007 to 2011. In soft X-rays LSI+61 303 was regularly observed by the PCA instrument on board of RXTE (one $\sim 2-3$ ks observation every 3-5 days) since Sept. 2007 up to Aug. 2011. During the Galactic plane scans and pointed observations LSI +61 303 was several times in the field of view of INTEGRAL imager ISGRI. In our analysis we use all publicly available data for the period from the January 2003 until October 2010. In the $\gamma$-ray band we use the data of Large Area Telescope (LAT) on board the FERMI satellite collected over the period from August 2008 to October 2011. The radio flux density was measured at $15.4 \mathrm{GHz}$ with the Ryle Telescope and its successor, the AMI Large Array (Cambridge UK). The Ryle Telescope used a bandwidth of $750 \mathrm{MHz}$, and the AMI uses $4 \mathrm{GHz}$. Observations are typically $30-60 \mathrm{~min}$ in duration.

Fig. 1 shows the $3-20 \mathrm{keV} \mathrm{X-ray} \mathrm{flux} \mathrm{from} \mathrm{the} \mathrm{source} \mathrm{as} \mathrm{a} \mathrm{function} \mathrm{of} \mathrm{the} \mathrm{orbital} \mathrm{and}$ superorbital phase (as seen by RXTE/PCA). From Fig. 1 one could see that the source exhibits on average one episode of increased X-ray activity per orbit. A regular increase of X-ray activity in the phase interval $\Delta \phi_{X} \simeq 0.2-0.4$ is accompanied by random variations of the source flux, with short flares appearing on the time scales $T_{\text {flares }, X} \ll \Delta \phi_{X} P_{\text {orb }}$. The average source flux in 3-20 keV energy range during the active/quiet part of the orbit indicated by the white dashed lines on Fig. 1 is about $1 \mathrm{mCrab} / 0.5 \mathrm{mCrab}$. Typical error of the flux measurment, $\sim 0.1 \mathrm{mCrab}$, is dominated by the uncertainity of the PCA background. 

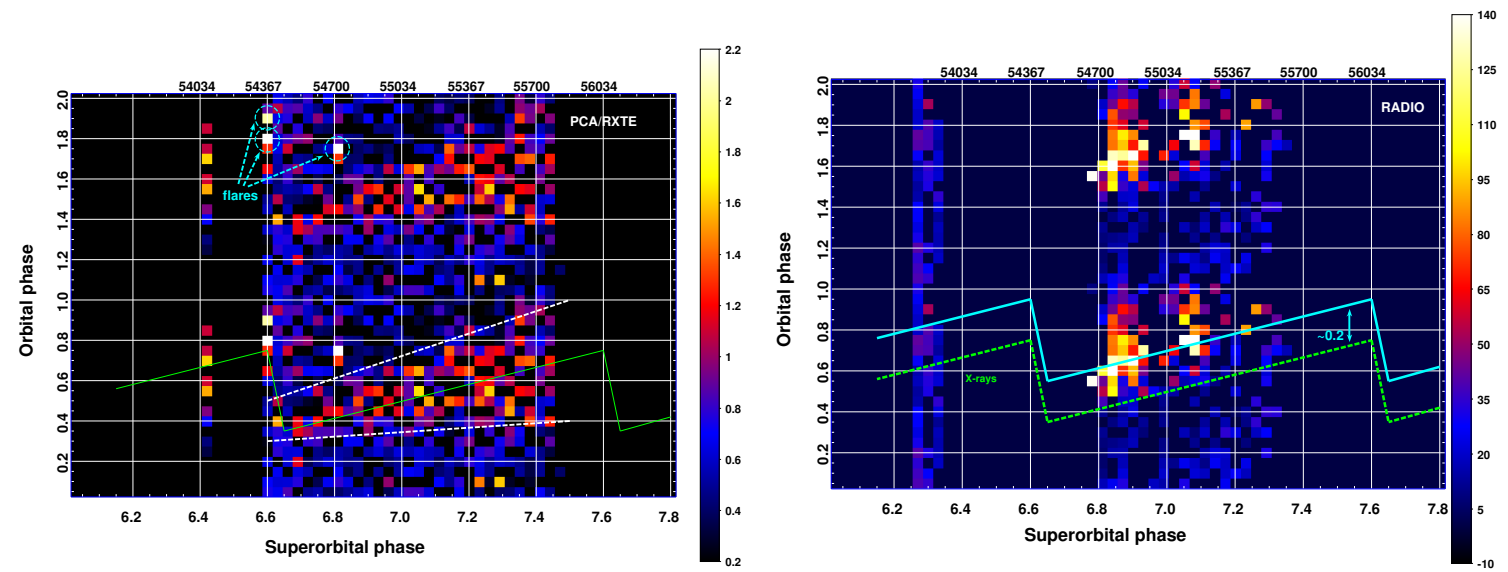

Figure 1: left:3 - $20 \mathrm{keV}$ flux from LSI +61 303. The color scale is expressed in mCrab units. right:Radio flux from LSI +61303 . The color scale is expressed in mJy units.
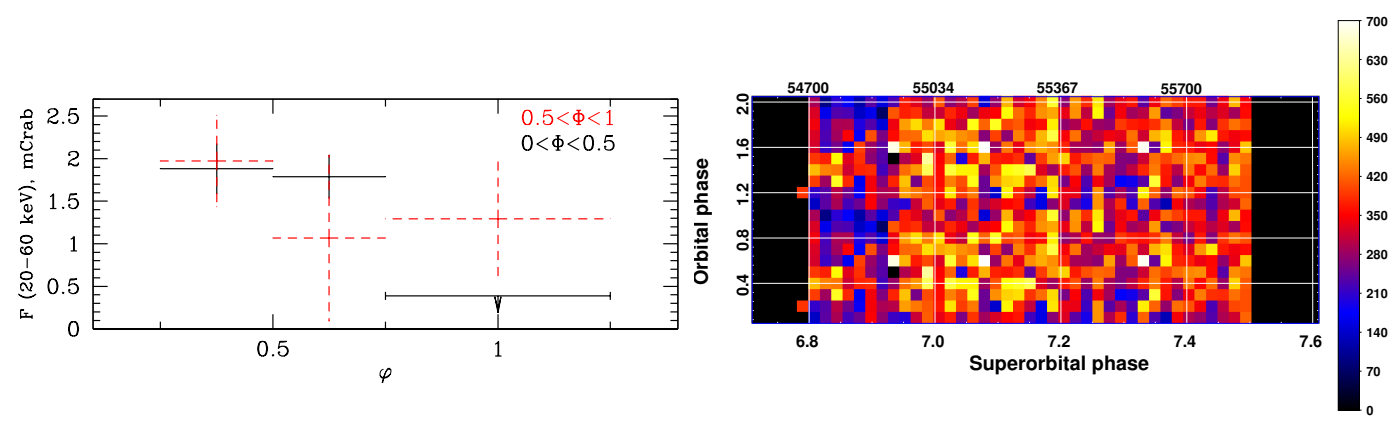

Figure 2: left: Averaged orbital variability of the hard X-ray flux (20 - $60 \mathrm{keV})$ from LSI +61 303Tंhe corresponding exposures (from left to right) are 525, 664 and $452 \mathrm{ksec}$ for the $0<\Phi<0.5$ and 298, 132 and $207 \mathrm{ksec}$ for the $0.5<\Phi<1$.right: Very high enregy $(\mathrm{E}>300 \mathrm{GeV})$ flux from LSI +61303 The color scale is expressed in mCrab units.

The average phase of the X-ray activity period $\phi_{X}$ varies on the superorbital time scale. From Fig. 1 we find that $\phi_{X}$ exhibits a systematic drift from $\phi_{X} \simeq 0.35$ to $\phi_{X} \simeq 0.75$ within one superorbital cycle. Such a drift is similar to the systematic drift of the phase of the periodic radio flares from $\phi_{R} \simeq 0.5$ to $\phi_{R} \simeq 1$ [5].

Evolution of the orbital variability of the source in hard X-rays $(20-60 \mathrm{keV})$ on the superorbital time scale, observed by INTEGRAL, is shown in Fig. 2. One could see that, similarly to the 3-20 keV range, the maximum flux happens during the orbital phase $0.25<\phi<0.5$ in the superorbital cycle phase $0.5<\Phi<1$. The maximum becomes wider and shifts toward $0.25<\phi_{X}<0.75$ in the superorbital phase $0<\Phi<0.5$.

Observations of the systematic drift of the phase of the radio flares from the source reported by [5] were performed several superorbital cycles before the X-ray monitoring campaign by RXTE. To verify the long-term stability of the range of the shifts of $\phi_{R}$ over many superorbital cycles we 
use the data of monitoring of the source in the radio band which are contemporaneous with the RXTE monitoring campaign. Right panel of Fig. 1 shows the radio flux of the source as a function of the orbital and superorbital phases. Comparing right panel of Fig. 1 with the equivalent figure from [5], we find that the overall drift pattern of the phase of the radio flare remained stable over several superorbital cycles. The same drift from the phase $\phi_{R} \simeq 0.55$ to $\phi_{R} \simeq 0.95$ is observed also in the radio data contemporaneous with the RXTE monitoring campaign.

Comparison of X-ray and radio superorbital variability patterns is shown in 1 . The average phase of the X-ray activity period always preceeds the phase of the radio flare by $\Delta \phi_{X-R} \simeq 0.2$, which corresponds to the time delay $\Delta T_{X-R}=\Delta \phi_{X-R} P \simeq 5.3 \mathrm{~d}$.

Contrary to the $\mathrm{X}$-ray and radio bands, the superorbital modulation pattern is not clearly visible in the $\gamma$-ray band. Right panel of fig. 2 shows the source flux in the $0.1-10 \mathrm{GeV}$ energy band plotted as a function of the orbital and superorbital phase, similarly to Fig. 1. Long-term source behaviour of the source in the $\mathrm{GeV}$ band is puzzling. Orbital modulation was clearly observable at the beginning of Fermi observations at the superorbital phase $6.8<\Phi<6.9$. The phase of the maximum orbital modulation of the $\mathrm{GeV}$ flux in this superorbital phase range was close to the phase of the X-ray activity. However, in the time period following the superorbital phase $\Phi \simeq 6.9$ a clear orbital modulation pattern disappeared.

\section{Discussion.}

A constant time delay between the drifting orbital phases of X-ray and radio flares could be naturally explained if one takes into account that radio and $\mathrm{X}$-ray emission originate from different regions. The radio emission is produced at large distance from the binary, $D_{R} \gtrsim 5 \times 10^{13} \mathrm{~cm}$ [13], while the X-ray flux is most probably produced at shorter distances of the order of the binary separation $3 \times 10^{12} \mathrm{~cm}<D_{X}<10^{13} \mathrm{~cm}$. Assuming that injection of high-energy electrons responsible for the X-ray and radio flares happens in the same event in the binary, one could attribute the time delay between the X-ray and radio flares to the time-of-flight of the high-energy particle filled plasma to the radio emission region. This gives an estimate of the plasma outflow velocity $v_{R} \simeq D_{R} / \Delta T_{X-R} \simeq 10^{8} \mathrm{~cm} / \mathrm{s}$, which is in good agreement with the asymptotic velocity of the stellar wind from the massive star in both the polar and equatorial regions [13].

The constant time delay between the X-ray and radio flare phases suggests the following scenario of production of the periodic radio flares. Once per orbit, an event of interaction of the compact object with the stellar wind leads to injection of high-energy particles into the stellar wind. The high-energy particles mixed into the stellar wind escape from the binary system with the stellar wind velocity. A radio flare occurs at the moment when the portion of the stellar wind filled with high-energy electrons reaches the distances $D \sim D_{R}$ at which the system becomes transparent to the radio waves.

Electrons producing synchrotron emission in the radio band have energies $E_{e} \simeq$ $10[B / 1 \mathrm{G}]^{-1 / 2}\left[v_{R} / 10 \mathrm{GHz}\right]^{1 / 2} \mathrm{MeV}$, where $v_{R}$ is the frequency of the radio synchrotron emission. The main cooling mechanisms for such electrons are synchrotron and/or inverse Compton emission with the characteristic cooling time scales $t_{S} \simeq 0.7[B / 1 \mathrm{G}]^{-3 / 2}\left[v_{R} / 10 \mathrm{GHz}\right]^{-1 / 2} \mathrm{yr}$ and $t_{I C} \simeq 6.5\left[D / 10^{13} \mathrm{~cm}\right]^{-2}$ d. Unless magnetic field in the system is much higher than $B \sim 10 \mathrm{G}$, the synchrotron and inverse Compton cooling time scales are not shorter than the time-of-flight from 
inside the binary orbit to the radio emission region. Thus energy losses do not prevent electrons injected in the binary system from traveling to the radio emission region on a time scale of several days.

The X-ray emission is most probably produced via the synchrotron mechanism by electrons of the energies $E_{e} \simeq 100[B / 10 \mathrm{G}]^{-1 / 2}\left[E_{X} / 5.4 \mathrm{keV}\right]^{1 / 2} \mathrm{GeV}$. This conjecture is supported by the observations of fast variability of X-ray emission on the time scales $t \sim 10 \mathrm{~s}$ [11] which is comparable to the synchrotron cooling time of $100 \mathrm{GeV}$ electrons in the $B \sim 10 \mathrm{G}$ magnetic field. The absence of any obvious break / cut-off features in the $\mathrm{keV}-\mathrm{GeV}$ source spectrum with a maximum in the $\sim 10-100 \mathrm{MeV}$ range is in favour of interpretation of the entire keV-GeV bump as a single spectral component. In such a model the $\mathrm{GeV}$ band emission is due to the synchrotron emission by the $10-100 \mathrm{TeV}$ electrons.

The phase of the $\gamma$-ray flare is close to the phase of the X-ray flare (Fig. 1 and right panel of 2 ), at least during a part of the superorbital cycle $6.8<\Phi<6.9$. It is natural to identify the phase of the X-ray/ $\gamma$-ray flare with the moment of formation of high-energy particle outflow inside the binary.

The phase of the X-ray activity is close to the phase of the periastron of the binary orbit $\phi_{\text {per }} \simeq 0.3$ at the superorbital phase $\Phi \simeq 0.5$ (Fig. 1) when the duration of the activity period is shortest. The phase of activity gradually shifts to the post-periastron interval $\phi_{X}>0.3$ over the superorbital period and almost reaches the phase of the apastron $\phi_{a p} \simeq 0.8$ toward the end of the superorbital cycle so that the X-ray emission is always delayed with respect to the apastron. Shift of $\phi_{X}$ is accompanied by the increase of the width of activity period.

A possible explanation for such behaviour could be found in a scenario in which the $4.6 \mathrm{yr}$ superorbital cycle is interpreted as the cycle of gradual buildup and decay of the equatorial disk of the Be star. At $\Phi \simeq 0.5$ the equatorial disk is weak. The phase of the closest encounter between the disk and the compact object is the phase of the periastron. Interaction of the compact object with the disk perturbs the disk and strips away a part of the disk which escapes from the system to the radio emission region. Gradual buildup of the equatorial disk due to ejection of matter from the Be star leads to the increase of the disk density and/or disk size. Such a scenario implies that extended radio emission shouldn't have a clear jet-like morphology, but rather have an irregular morphology varying over the orbital and superorbital cycle.Such a variable morphologu is indeed observed in the radio band [4].

The shift of the phase $\phi_{X}$ of ejection of a portion of the disk could be explained by the increase of the time of accumulation of energy sufficient for ejection. The kinetic energy needed to strip away a part of the disk is comparable to the gravitational binding energy of the disk, $U \sim$ $G_{N} M_{*} \rho_{d} R_{d} H_{d} \sim 10^{40}\left[\rho_{d} / 10^{13} \mathrm{~g} \cdot \mathrm{cm}^{-3}\right]\left[R_{d} / 10^{12} \mathrm{~cm}\right]\left[H_{d} / 10^{12} \mathrm{~cm}\right] \times\left[M_{*} / 10 M_{\odot}\right] \mathrm{erg}$, where $\rho_{d}, R_{d}$ and $H_{d}$ are the density, radius and thickness of the equatorial disk. Such energy should be transmitted to the disk by the compact object at each disk-compact object interaction event. Suppose that the compact object injects energy in the disk at a constant rate $P \mathrm{erg} / \mathrm{s}$ at each interaction event. The energy sufficient for ejection of a part of the disk is then accumulated on a time scale $T_{e j} \sim U / P \sim 1\left[P / 10^{35} \mathrm{erg} / \mathrm{s}\right]^{-1} \mathrm{~d}$. Accumulation of mass in the disk leads to the increase of $T_{e j}$ and, as a consequence, to the shift of $\phi_{X}$.

If the disk size reaches the size of the binary orbit, the compact object always moves inside the disk and continuously perturbs it. Studies of the high-mass X-ray binaries with Be stars show 
that in this case the compact object induced instabilities in the disk might lead to destruction and complete loss of the disk. Our hypothesis is that the loss of the disk corresponds to the period in the superorbital phase range $\Phi \simeq 0.4-0.5$ when the strength of X-ray and radio flares decreases and no systematic periodic variability of the source is observed (see Fig. 1 and [5]). Ejection of matter from the equatorial regions of Be star leads to formation of a new disk at around $\Phi \simeq 0.5$ and a new cycle of disk growth/decay starts.

In such a scenario, regularity of the superorbital modulation in the system could be readily explained. The constant growth rate of the equatorial disk of Be star is determined by the stable rotation of the Be star. The period of superorbital modulation is determined by the fixed time scale on which the disk growth to the size comparable to the size of the binary orbit. This scenario for the origin of the orbital and superorbital modulations of the source flux could be tested using the $\mathrm{H} \alpha$ data which provide a diagnostic of the state of the equatorial disk of Be star [12, 9]. Growth and decay cycles of the disk lead to the variations of the overall strength and shape of the H $\alpha$ line. Variability of the line intensity and profile on the time scale of the superorbital modulation was demonstrated by [12]. Monitoring of the $\mathrm{H} \alpha$ line on the time scale of several superorbital cycles would show if the the observed variability corresponds to the periodic buildup and decay of the disk. Periodic ejection of a part of the disk as a result of the compact object-disk interaction might be responsible for occurence of transient red or blue "shoulders" of $\mathrm{H} \alpha$ line as observed by [9]. Systematic re-observation of the repetition of occurence of the shoulders in many orbital cycles and correlation of the phase of occurence of the shoulders with the phases of X-ray flares would provide a direct test for our model.

\section{References}

[1] Abdo A.A. et al., 2009, Ap.J., 701, L123.

[2] Chernyakova M., Neronov A., Walter R., 2006, MNRAS, 372, 1585.

[3] Chernyakova M., Neronov A.,Molkov S., Malyshev D., Lutovinov A., Pooley G., 2012, Ap.J., 747, L29.

[4] Dhawan V., Mioduszewski A., \& Rupen M. 2006, PoS, Proceedings of the VI Microquasar Workshop: Microquasars and Beyond, 52.1

[5] Gregory P.C., 2002, Ap.J., 575, 427.

[6] Li, J., Torres, D. F., Zhang, S., et al. 2012, ApJ, 744, L13

[7] Massi M., et al., 2004, A\&A, 414, L1.

[8] McSwain M.V., et al., 2011, Ap.J. 738, 105.

[9] McSwain M.V. et al., 2010, Ap.J., 724, 379.

[10] Paredes J.M., Massi M., Estalella R., Peracaula M., 1998, A\&A, 335, 539.

[11] Smith A., et al., 2009, Ap.J., 693, 1621.

[12] Zamanov R.K., et al., 1999, A\&A, 351, 543.

[13] Zdziarski A.A., Neronov A., Chernyakova M., 2010, MNRAS, 403, 1873. 